PHYSICAL REVIEW D 93, 089902(E) (2016)

\title{
Erratum: Backreaction of particle production on false vacuum decay [Phys. Rev. D 93, 025024 (2016)]
}

\author{
Cyril Lagger \\ (Received 21 March 2016; published 5 April 2016)
}

DOI: 10.1103/PhysRevD.93.089902

According to [1], we noticed that the prefactor $C$ in Eq. (17) is not a constant but a $\tau$-dependent $c$ number $C(\tau)$. Thus, Eq. (17) should read as

$$
|\phi(\tau)\rangle=\mathcal{X}_{\tau}\left|O_{\tau}\right\rangle=C(\tau) \exp \left(\frac{1}{2} \sum_{\alpha, \beta} D_{\alpha \beta}(\tau) a_{\alpha, \tau}^{\dagger} a_{\beta, \tau}^{\dagger}\right)\left|O_{\tau}\right\rangle .
$$

Consequently, footnote 7 must be omitted, and the prefactor $|C(0)|^{2}:=|C(\tau=0)|^{2}$ has to be inserted in both Eqs. (36) and (51) as follows:

$$
\frac{\Gamma_{\mathrm{Ba}}}{\mathcal{V}}=\frac{\hat{\rho}_{r}(0)}{\hat{\rho}_{r}(-\infty)}=|C(0)|^{2} e^{-S_{B}} \frac{\mathcal{F}(0)}{\mathcal{F}(-\infty)},
$$

and

$$
\frac{\Gamma_{\mathrm{Ba}}}{\mathcal{V}}=\frac{|C(0)|^{2} e^{-S_{B}}}{\sqrt{\operatorname{Det}\left(\mathbb{1}-D_{\tau=0}^{2}\right)}}=|C(0)|^{2} e^{-S_{B}-\frac{1}{2} \operatorname{Tr} \ln \left(1-D_{\tau=0}^{2}\right)}
$$

As explained in [1], this prefactor $|C(0)|^{2}$ can be evaluated from the path integral method of Callan and Coleman [2]. It corresponds to the subleading effect of one-loop quantum fluctuations of the field $\phi$ on the decay probability of the field $\sigma$.

On the other hand, the backreaction of the real $\phi$ particles on the decay rate of $\sigma$ is not modified by the above correction and is still given by the expression $\left[\operatorname{Det}\left(\mathbb{1}-D_{\tau=0}^{2}\right)\right]^{-1 / 2}$. Therefore, our interpretation of the backreaction of particle production and its effects on the semiclassical decay rate $e^{-S_{B}}$ remain unchanged.

[1] V. A. Rubakov, Nucl. Phys. B245, 481 (1984).

[2] C. G. J. Callan and S. R. Coleman, Phys. Rev. D 16, 1762 (1977). 